\title{
La cumulativité du savoir sociologique entre mythe et réalité
}

Jacques Coenen-Huther

\section{OpenEdition}

Édition électronique

URL : http://journals.openedition.org/ress/363

DOI : $10.4000 /$ ress.363

ISSN : 1663-4446

Éditeur

Librairie Droz

Édition imprimée

Date de publication : 1 février 2005

Pagination : 23-33

ISBN : 2-600-00958-2

ISSN : 0048-8046

Référence électronique

Jacques Coenen-Huther, «La cumulativité du savoir sociologique entre mythe et réalité », Revue européenne des sciences sociales [En ligne], XLIII-131 | 2005, mis en ligne le 04 novembre 2009, consulté le 20 avril 2019. URL : http://journals.openedition.org/ress/363 ; DOI : 10.4000/ress.363 
Jacques COENEN-HUTHER

\section{LA CUMULATIVITÉ DU SAVOIR SOCIOLOGIQUE ENTRE MYTHE ET RÉALITÉ}

Pour se traduire en un projet scientifique crédible, l'intention de science qui a donné naissance à la sociologie implique la constitution graduelle d'un savoir cumulatif. Nombreux sont les sociologues pour qui ce critère de scientificité ne semble guère problématique, soit qu'ils tiennent l'accumulation régulière de connaissances pour un fait acquis, soit que leur pratique de recherche ne les conduise pas à s'interroger à ce sujet. Force est pourtant bien de constater que les travaux de plusieurs générations de sociologues n'ont que très faiblement répondu à cette exigence majeure. Les symptômes d'un indéniable défaut de cumulativité ne manquent pas. Examinons-les ici.

\section{L'AMBIVALENCE À L'ÉGARD DES CLASSIQUES}

La communauté sociologique reste de toute évidence partagée entre le désir de prendre appui sur une tradition philosophique de réflexion sur la condition humaine remontant à l'Antiquité grecque et la volonté de ne s'inspirer que de l'état le plus récent des connaissances en sciences humaines. C'est ce que Robert Merton, qualifiait de dilemme de l'érudition et de l'originalité (1949, 1968, p. 30). Ni l'une ni l'autre de ces orientations ne favorise une attitude propice à l'élaboration d'un savoir cumulatif. La mise en évidence d'une longue tradition de réflexion présociologique consubstantielle à la pensée occidentale depuis l'Antiquité contribue à rendre floue la frontière entre philosophie sociale et sociologie. Il se crée ainsi un climat intellectuel de scepticisme à l'égard de la notion même de progrès possible dans notre connaissance des mécanismes de la vie en société. Au cours de sa leçon inaugurale, Georges Goriely, professeur de sociologie à Bruxelles, mettait en doute «que la lecture de n'importe quel ouvrage qui s'intitule sociologie» soit plus fructueuse que celle de la République de Platon ou de la Politique d'Aristote (1950, p. 26). A tort ou à raison, cette opinion reste répandue un demi-siècle plus tard. Mais la volonté de marquer nettement la rupture entre la sociologie comme projet scientifique et sa longue préhistoire - ce que Goriely qualifiait de «prétention outrecuidante» (ibid., p. 33) - n'est paradoxalement guère plus favorable à la cumulativité des connaissances sociologiques. Elle suscite des réactions de condescendance à l'égard des classiques de la discipline et tend à rejeter régulièrement dans l'oubli les travaux des prédécesseurs alors qu'aucun progrès discernable ne se manifeste dans l'intelligence des faits. C'est ce que Sorokin qualifiait de «complexe de l'inventeur», y voyant une «espèce d'amnésie » à l'égard des «acquisitions antérieures » et une prétention peu justifiée à l'originalité (1956, 1959, p. 7). 
L'attitude «érudite»- celle qui se tourne volontiers vers les œuvres du passé - est elle-même chargée d'ambiguïté. Il arrive certes qu'on cherche dans les travaux des «pères fondateurs» des hypothèses à vérifier dans un contexte nouveau, à l'aide de techniques d'analyse plus affinées, mais c'est relativement rare. Une telle démarche, accomplie systématiquement, devrait logiquement conduire à un usage de plus en plus sélectif des travaux en question. Mais ce qui se manifeste le plus souvent est une forme de déférence hyperbolique, suggérant implicitement, ou explicitement à l'instar de Nisbet, que l'âge d'or de la sociologie est derrière nous (Nisbet, 1966, 1993, p. 89). Merton, par ailleurs adepte d'une conception résolument scientifique de la sociologie ${ }^{1}$, traduisit clairement ce sentiment de se trouver confronté à des accomplissements intellectuels inégalés: «More than one sociologist», écrit-il, «has had the self-deflating experience of finding that his independant discovery is unwittingly a rediscovery, and, moreover, that the language of the classical prediscovery, long lost to view, is so crisp, so eloquent, or so implicative as to make his own version only second-best » (1949, 1968, pp. 35-36). De façon plus générale, Merton et Parsons décrivent le rapport aux classiques en des termes qui évoquent une relation de type littéraire davantage qu'un processus cumulatif de production de science. Pour le premier, lorsque l'ouvrage d'un auteur classique mérite d'être lu, il mérite de l'être périodiquement car ce qui est ainsi communiqué se modifie à la suite de l'interaction entre l'auteur défunt et le lecteur: «Just as the Song of Songs is different when it is read at age 17 and at age 70, so Weber's Wirtschaft und Gesellschaft or Durkheim's Suicide or Simmel's Soziologie differ when they are read at various times » (ibid., pp. 36-37). Le second exprime une idée semblable lorsqu'il écrit que «without an intellectually rich tradition to revisit, I do not think that disciplines like our own, with all their difficulties and complexities, could advance. That is the basis for my plea ... for our continuing to revisit the classics » (1981, p. 193, mots soulignés par JCH).

\section{L'INSTRUMENTALISATION DES CLASSIQUES}

Les manifestations de dévotion pour les œuvres classiques ont toutefois acquis une fonction bien particulière dans la sociologie moderne, même s'il s'agit d'un de ces tabous qu'on n'évoque que dans les conversations privées. L'invocation d'un auteur du passé fait incontestablement partie des procédés d'argumentation couramment utilisés pour légitimer un point de vue théorique actuel. Cette instrumentalisation des classiques ne va d'ailleurs pas sans une certaine désinvolture à l'égard de la vérité historique. Marx et Tocqueville sont consacrés de façon posthume "pères fondateurs» de la sociologie. Cette reconnaissance est pleinement justifiée par leur apport à la pensée sociale mais elle tend à faire oublier que Marx n'utilisa jamais le terme «sociologie» alors qu'il le connaissait et que Tocqueville, publiciste comme le $\mathrm{XIX}^{\mathrm{e}}$ siècle en connut beaucoup, avait le souci d'être entendu par l'élite mondaine des salons parisiens et non d'être admis dans quelque

Et qui n'hésita pas à placer en exergue d'un de ses articles une citation de Whitehead selon laquelle «A science which hesitate to forget its founders is lost » (Merton, 1949, 1968, p. 1). 
communauté scientifique (Coenen-Huther, 1997, p. 4). Pour mieux justifier l'abandon d'un positivisme daté, les conceptions méthodologiques de Max Weber sont gratifiées a posteriori d'une originalité qu'elles n'avaient pas tout à fait, étant avant tout réactives dans l'environnement intellectuel du moment (Tenbruck, 1959). Un Durkheim souvent caricaturé est tantôt le repoussoir de l'œuvre weberienne, tantôt le propagateur inconscient de préceptes weberiens ${ }^{2}$. Reflux de la weberomania et retour à la tradition durkheimienne: on fait observer que le type idéal weberien ne comporte aucune innovation de sens par rapport à l'usage durkheimien du terme (Coenen-Huther, 2003, p. 532).

L'instrumentalisation des classiques contribue à créer un climat intellectuel où une forme subreptice d'argument d'autorité tend à remplacer - ou au moins à renforcer - une démonstration. Invoquer Marx n'est actuellement plus de bon ton mais assortir son argumentation de références à d'autres classiques aide de toute évidence à obtenir l'adhésion de son auditoire. Au gré de modes intellectuelles que les différentes écoles ne manquent pas de stimuler, c'est Durkheim, Pareto, Simmel, Tocqueville ou Weber qui sera appelé à épauler le raisonnement proposé. Comte, il est vrai, n'a plus guère la cote en ce moment. Le recours systématique aux citations de grands ancêtres peut également relever de la prudence tactique. A l'heure où la force de l'argumentation rationnelle est ébranlée par le relativisme ambiant, illustrer son propos par un exemple extrait d'un ouvrage canonique est moins périlleux que prendre l'actualité à bras-le-corps. Non que les classiques échappent entièrement au travail de sape de la pensée relativisante, mais ils suscitent généralement moins de réactions émotionnelles que l'évocation de thèmes actuels. Une autre conséquence de cette instrumentalisation est que des enseignements d'histoire de la sociologie tiennent fréquemment lieu d'enseignements de théorie générale, donnant ainsi l'impression qu'il s'agit avant tout de propager des vérités révélées. Dans l'ensemble des sciences humaines, la sociologie est probablement la discipline au sein de laquelle l'esprit scolastique est le plus répandu.

\section{LES INNOVATIONS DE LANGAGE}

Comme j'ai eu l'occasion de le rappeler récemment (Coenen-Huther, 2004b, p. 241), les innovations en sociologie sont souvent des innovations de vocabulaire. Ceci non plus n'est pas favorable à la cumulativité du savoir sociologique car ceux qui se donnent des airs d'originalité «en recyclant des idées anciennes à l'aide d'une réthorique nouvelle» (Dubois, 1999, p. 2) ont bien évidemment intérêt à être aussi discrets que possible à ce sujet. Evoquons ici un exemple très actuel. C'est depuis l'avènement de la modernité que l'individu libéré des vieux liens communautaires se caractérise par la diversité des contextes d'interactions au sein desquels il est appelé à se mouvoir et la pluralité des rôles qu'il lui appartient d'assumer. Depuis une quinzaine d'années est apparue dans la littérature sociologique l'expression «individu pluriel» qui témoigne de la perméabilité du

On consultera à ce sujet le livre récent de Laurent Mucchielli, Mythes et histoire des sciences humaines (2004) ainsi que l'ouvrage dirigé par Monique Hirschhorn et Jacques Coenen-Huther, Durkheim et Weber. Vers la fin des malentendus? (1994), auquel Mucchielli se réfère (2004, pp. 3136). 
champ de la sociologie aux influences médiatiques mais qui n'apporte rien de neuf à la conceptualisation de l'individu moderne à l'identité multiple et fluctuante. Il pourrait ne s'agir que d'une nouvelle habitude de langage sans conséquence si l'on n'avait voulu y voir la marque d'une situation sociale inédite (Lahire, 1998) et en faire l'objet de «nouvelles sociologies » (Corcuff, 1995) dont la nouveauté est plus que discutable. Il est vraisemblable que la littérature centrée sur l'acteur dit «pluriel» n'aurait pas connu un tel essor si la théorie des rôles, pourtant très efficace pour analyser les situations créées par la fragmentation du social, n'avait été relativement délaissée depuis un quart de siècle. La prétention à la nouveauté y gagne, l'accumulation de connaissances y perd.

Il arrive qu'on justifie l'introduction d'une innovation terminologique par la nécessité d'insérer une notion dans un cadre théorique nouveau. Ainsi en est-il de l'usage extensif que Pierre Bourdieu a fait de la notion de «capital» (capital économique, culturel, politique, scolaire, social, symbolique) et en particulier du terme de «capital social». Mis en correspondance avec les termes de «capital culturel» et de «capital économique», ce terme permet la mise en évidence de symétries ou d'asymétries entre les différentes sortes de capital. Pour s'exprimer dans la langage de Bourdieu, il permet de définir «la position occupée dans l'espace social, c'est-à-dire dans la structure de la distribution des différentes espèces de capital» (Bourdieu, 1994, p. 28). De cette façon, le terme véhicule aussi l'idée d'accumulation intergénérationnelle. Autrefois, des raisonnements semblables à ceux qu'a présentés Bourdieu étaient formulés à l'aide d'une terminologie moins homogène et notamment à l'aide des termes de «ressources» ou de «talents sociaux». Sans doute, le langage proposé par Bourdieu introduit explicitement une analogie économique ainsi qu'une distinction entre l'héritage et l'acquisition, ce qui peut être considéré comme un perfectionnement terminologique. Mais il crée aussi un sentiment peu justifié de nouveauté et de rupture avec les travaux antérieurs, qui sert davantage la satisfaction intellectuelle et le prestige de son auteur que la cumulativité du savoir sociologique.

\section{LES CRISES DE LA SOCIOLOGIE}

La communauté sociologique est régulièrement agitée par des crises qu'on qualifie un peu abusivement de «révolutions scientifiques» depuis que s'est propagé parmi les sociologues l'ouvrage de Thomas Kuhn, The Structure of Scientific Revolutions (1962). Le remplacement d'un paradigme dominant par un autre est un épisode non cumulatif (ibid., 1962, 1970, p. 92). Il ne résulte pas d'une accumulation de connaissances mais d'une nouvelle manière d'organiser et d'interpréter les connaissances disponibles. La genèse du fonctionnalisme sociologique moderne a paru s'accorder plus ou moins avec le modèle kuhnien ${ }^{3}$. Le fonctionnalisme a semblé en effet marquer une rupture épistémologique avec l'état antérieur de la théorie sociologique. Avec son ascension intellectuelle et institutionnelle, la sociologie semblait passer d'une phase préparadigmatique,

Pour un exposé de l'état de la question tel qu'il se présentait il y a un quart de siècle, voir mon livre Le fonctionnalisme en sociologie : et après? (1984, chap. III). 
caractérisée par la multiplicité des points de vue en présence (ibid., p. 163), à une phase pluriparadigmatique marquée par la compétition de quelques paradigmes importants. Loin de resserrer la compétition, le déclin du fonctionnalisme - déclin non réductible à une évolution interne à la théorie sociologique - a laissé le champ libre à la résurgence de courants théoriques antérieurs qui ne manquèrent pas d'être salués comme des innovations dans le climat général d'amnésie intellectuelle fustigé par Sorokin.

Depuis lors apparaissent régulièrement des thèmes de discussion dont la prétention à l'originalité n'est rendue crédible que par l'oubli dans lequel tombent rapidement les travaux des prédécesseurs. Le $17^{\circ}$ congrès de l'Association Internationale des Sociologues de Langue Française (L'individu social. Autres réalités, autres sociologies? Tours, 5-9 juillet 2004) en offre un exemple presque caricatural. Un siècle et demi après les Thèses sur Feuerbach, trois quarts de siècle après la parution de Mind, Self and Society de Georges Herbert Mead, les congressistes furent invités à prendre conscience du fait que l'individu est un «être social» et à en tirer les conclusions. Dans le domaine des sciences sociales, il n'est guère d'autre discipline à vocation scientifique qui se complaise ainsi dans la répétition fastidieuse de débats qui n'ont plus leur raison d'être. Mais la charte du congrès proposée par le comité organisateur comportait deux aspects distincts, aussi peu favorables l'un que l'autre à l'accumulation de connaissances: tout d'abord le sentiment que le caractère social de l'individu - idée fondatrice de la sociologie s'il en est - devait absolument être réaffirmé à nouveaux frais; ensuite l'idée qu'une réalité nouvelle, marquée par une fragmentation accrue de l'espace social et une plus grande fluidité des affiliations, devait nécessairement conduire à «repenser la sociologie». Il convient, certes, de faire ici la part de ce qui est propre à la littérature de congrès. Mais qu'une conjoncture sociale et politique nouvelle appelle nécessairement la mise en place d'un appareil conceptuel nouveau est une idée typique d'une discipline peu à même de constituer un savoir cumulatif.

\section{LES SOCIOLOGUES ET LEUR PARCOURS DE VIE}

Si l'on examine le parcours de vie de sociologues ayant atteint une certaine notoriété, on constate que la renommée s'acquiert plus souvent par la présentation d'un cadre conceptuel nouveau que par l'application de catégories conceptuelles éprouvées à un objet nouveau. Il n'est d'ailleurs pas rare que les nouveautés ainsi proposées fassent l'objet d'une désaffection notable après le décès de leurs auteurs. L'œuvre de Georges Gurvitch et celle de Talcott Parsons en fournissent des exemples frappants, celle du premier plus encore que celle du second. Dans les années trente, Parsons se demandait «Qui lit encore Spencer?» (1937). A l'heure actuelle, on pourrait malheureusement s'interroger de la même façon à son sujet et se demander si The Social System conserve beaucoup de lecteurs dans la jeune génération.

Mais si la sociologie a une histoire, chaque sociologue a son histoire. Et la cohérence qu'on peut y introduire est toute relative. Il est assez fréquent que la carrière de sociologues de renom soit marquée par l'une ou l'autre rupture que n'explique nullement l'accumulation de connaissances, rendant ainsi plus difficile la constitution progressive d'un canon de méthode pouvant réaliser l'ac- 
cord des esprits et faire l'objet d'une transmission intergénérationnelle. C'est une tranche de vie et, on peut le supposer, un long cheminement intellectuel, qui séparent le Boudon des années soixante, célébrant la «révolution durkheimienne » en matière d'analyse causale (1967) du Boudon des années quatre-vingts, reléguant l'analyse causale en note - une très longue note, il est vrai - pour ne plus lui assigner qu'une fonction heuristique $(1986 \text {, chap. 10, note } 11, \text { p. } 313)^{4}$. On a pu noter une évolution semblable chez Parsons. Après une première tentative de synthèse paradigmatique dans les années trente (1937), celui-ci se fera plus durkheimien à la fin des années quarante (1951) pour se rapprocher à nouveau de Weber à la fin des années soixante (1968). Moins fondamental mais plus spectaculaire fut le revirement de Pierre Bourdieu qui, dans La misère du monde, prit ses distances à l'égard de pratiques d'enquête qui avaient longtemps été les siennes et déclara ne plus y voir que «la volonté de mimer les signes extérieurs de la rigueur des disciplines scientifiques les plus reconnues» (1993, p. 1389).

Chez plus d'un membre éminent de la communauté sociologique arrivé en fin de carrière, on a pu noter des propos désabusés à l'égard d'une discipline qui ne semble pas tenir ses promesses de scientificité. L'histoire de la sociologie se double ainsi d'une tradition de critique de la sociologie qui porte notamment sur les modes de régulation du débat scientifique entre sociologues. De Max Weber à Irving Horowitz en passant par Charles Wright Mills, Peter Berger et Ralf Dahrendorf, il y a une incontestable continuité que prolonge notre éminent collègue Giovanni Busino (Coenen-Huther, 1995, p. 185). Ce dernier signale l'absence des conditions minimales permettant l'élaboration collective d'un savoir cumulatif lorsqu'il exprime son regret de voir «la communauté sociologique éclatée, les échanges et les communications interrompus ...», les sociologues ne se passionnant plus «que pour les produits de leur propre chapelle» (1993, pp. 9$11)$.

L'esprit sociologique exige cependant qu'on aille au-delà du constat douloureux d'une situation qui ne prédispose pas à l'enthousiasme. Essayons, de façon certainement non exhaustive, d'esquisser les causes de cet état de fait.

\section{UN OBJET QUI SE TRANSFORME}

Il ne serait pas impossible de définir l'objet de l'investigation sociologique d'une façon qui lui confère la stabilité nécessaire à la cumulativité des connaissances. Il faudrait pour cela orienter la sociologie vers la recherche d'invariants du comportement humain qui trancendent les époques et les cultures. De tels invariants ne peuvent surgir que du raisonnement sur les formes de l'interaction sociale, selon le projet hérité de Simmel, ou de la mise en lumière de constantes de l'esprit humain, comme nous y invite Claude Lévi-Strauss. La mise en œuvre résolue d'un tel programme de recherche pourrait réintroduire à un certain niveau la notion de loi en sociologie ou au moins y faire prospérer l'usage de propositions structurelles ou conditionnelles du type « $\mathrm{Si} \mathrm{A}$, alors B ». La sociologie se rappro-

4 Je reprends ici une remarque formulée dans mon mémoire d'habilitation Sortir du labyrinthe? Pour une cumulativité du savoir sociologique: illusions et réalités (Toulouse II, 1991). 
cherait alors du statut de discipline scientifique dont elle ne bénéficie en ce moment «qu'en vertu d'une fiction sémantique et d'une espérance philosophique à laquelle les confirmations manquent encore » (Lévi-Strauss, 1973, p. 341).

Une telle réorientation, en rupture totale avec les tendances dominantes, ne paraît cependant guère s'annoncer. Quels que soient les domaines de spécialisation, les thèmes de recherche sont loin d'être définis à un tel niveau d'abstraction et de généralité. Le discours sociologique en devient plus accessible au profane mais il est confronté à des réalités beaucoup plus fluctuantes dans le long terme, voire dans le moyen terme. Le fossé qui sépare la sociologie des sciences de la nature est alors béant: «Il n'existe, il n'a jamais existé qu'un seul monde physique dont les propriétés sont demeurées les mêmes en tous temps et en tous lieux, pendant qu'au cours des millénaires et de-ci de-là, n'ont cessé de naître et de disparaître, comme un papillotement éphémère, des milliers de mondes humains » (Lévi-Strauss, ibid. p. 364). Qu'il s'agisse de travaux sur la famille, sur les organisations, sur la médecine et la santé, sur les religions, sur la science, etc, les réalités étudiées se sont si profondément transformées en un demi-siècle qu'elles défient la volonté de constituer un savoir cumulatif dans la mesure où elles semblent rendre très rapidement obsolètes les résultats de recherche et, avec eux, les modes d'analyse qui ont permis de les présenter. Cette situation est aggravée par la confusion qui entoure les procédés de modélisation en sociologie. A la différence des modèles économiques dont une fonction reconnue est de mesurer les écarts avec la réalité empirique, les modèles sociologiques suscitent de la méfiance dès qu'ils s'écartent un tant soit peu de la réalité observable. Ainsi l'analyse parsonienne de la relation patient-médecin (Parsons, 1951, chap. 10) est fondée sur le concept de «rôle du malade» (sick role) défini par une série d'attentes institutionnalisées, assorties de sanctions sociales (ibid., p. 436). Le «rôle du malade» est à la fois un concept analytique et une catégorie descriptive. Comme catégorie descriptive, il a fait l'objet de nombreuses critiques en raison des limites de son domaine d'application et de l'évolution de la pratique médicale. Mais on lui a également reproché de reposer sur une vision trop généralisée de la maladie et de ne pas rendre compte de la grande variété des attitudes, liées notamment à la gravité de la condition et à la stigmatisation qui s'attache à certains états. Ces critiques, malgré leur pertinence, ont dépassé leur objet. On a ainsi largement perdu de vue que la présentation idéal-typique de l'articulation entre le rôle du malade et celui du médecin dans une structure de rôles complémentaires conserve une valeur de modèle et peut être utilisée pour analyser tous les écarts par rapport au modèle. Dans une période de changement accéléré dans tous les domaines, la confusion entre la modélisation et la description est certainement un frein à la cumulativité du savoir sociologique.

\section{LE CERCLE PIAGÉTIEN}

Quelles que soient les conclusions qu'il en tire, le sociologue n'échappe pas à la circularité de la relation entre le sujet connaissant et l'objet de sa connaissance. A cette relation circulaire s'est attaché le nom de Piaget (1967, p. 1173). Appliqué aux sciences sociales, le cycle piagétien sujet-objet rend compte du fait que «le chercheur fait lui-même partie de la société qu'il se propose d'étudier et qui joue 
un rôle prépondérant dans l'élaboration de ses catégories intellectuelles (Goldmann, 1967, p. 992). On débouche ainsi sur ce que Jean-Michel Berthelot qualifie de «dialogique de la connaissance» non seulement psychologique mais également sociétale, à savoir une confrontation entre « une matrice d'événements et de corrélations concrètes, et un point de vue disciplinaire» (1996, p. 155). Pour Berthelot, les «saillances» émergeant de la matrice des faits observables et les «pertinences » introduites par le point de vue disciplinaire sont les deux faces de toute construction cognitive (ibid., p. 156). Mais le cercle piagétien ne peut que faire intervenir la succession des générations et les étapes du parcours de vie dans le mode de développement de la connaissance sociologique. Il n'est pas indifférent que l'observateur de la réalité sociale ait vingt ans ou qu'il en ait soixante, qu'il n'ait derrière lui que les illusions de l'adolescence ou au contraire l'expérience accumulée de la personne d'âge mûr. Et il est sans doute inévitable qu'à un rythme correspondant à la succession des générations (Piaget, 1965, p. 50), des illusions se répètent, des erreurs renaissent, des voies sans issue soient empruntées à nouveau. Certains acquis de la connaissance sociologique paraissent trop directement induire un scepticisme désabusé pour n'avoir pas régulièrement à céder devant les enthousiasmes volontaristes. D'autres s'opposent trop nettement à la réalité vécue de tout un chacun pour pouvoir faire l'objet d'une transmission sans problème. Chaque génération doit non seulement comprendre, expliquer, démontrer mais aussi convaincre. Et cette double nature de l'argumentation sociologique ne peut qu'être un frein puissant à toute accumulation intergénérationnelle de connaissances. Entre les « saillances » de l'objet et les «pertinences» du sujet s'insère un principe de sélectivité issu des caractéristiques de l'auditoire constituant la cible de l'argumentation. Dès lors, un faisceau de variables contribue à situer et à dater le processus de constitution de connaissance: variables liées à l'objet, variables liées au sujet, mais aussi variables correspondant aux caractéristiques de l'auditoire. Le dialogue s'établit ainsi plus souvent avec les contemporains sociologues ou non - qu'avec les générations précédentes de sociologues.

\section{LES MODES INTELLECTUELLES}

Il fut un temps où le succès médiatique ou mondain ne contribuait guère à la reconnaissance d'un sociologue par ses pairs ${ }^{5}$. De nos jours, au contraire, la notoriété acquise auprès d'un large public cultivé accroît le prestige et l'influence de ceux qui l'acquièrent, même sur le plan strictement académique. La médiatisation transforme la «compétition des paradigmes » en phénomènes de modes intellectuelles. Un exemple frappant fut le succès rapide du structuralisme à la fin des années 60 et son reflux tout aussi rapide quelques années plus tard. Tant le succès initial que le reflux ont obéi à des effets de mode largement indépendants des controverses scientifiques portant sur l'anthropologie structurale de Claude LéviStrauss. L'impact énorme des médias a notamment pour conséquence que des auteurs ayant acquis les faveurs du «grand public» contribuent plus largement

J'ai développé ceci dans mon article The Paths of Recognition: Boudon, Bourdieu, and the Second Market of Intellectuals (2000) ainsi que dans ma Sociologie des élites (2004a, pp. 20-22). 
qu'autrefois à structurer le débat scientifique. Les thèmes de colloques et de congrès en portent la marque. On peut, à la manière de Bourdieu (1996), présenter la situation qui s'est ainsi créée comme le résultat de la compétition entre des domaines professionnels dominés par des normes de comportement différentes: le «champ scientifique» et le «champ journalistique». Ce dernier a augmenté démesurément son influence en raison d'une situation de monopole de la production et de la diffusion de l'information; il est à présent en mesure de pénétrer les autres champs - et d'y imposer de nouvelles normes - à l'aide d'une stratégie qui peut être qualifiée de stratégie «du cheval de Troie». Mais les différents «champs» n'ont pas tous le même degré d'autonomie. Celui-ci est maximal lorsque la discipline en question offre un marché clos où les «producteurs de connaissance» n'ont comme interlocuteurs que leurs propres collègues. Un champ très autonome peut résister à l'invasion du champ journalistique. A l'inverse, plus un champ est hétéronome, ouvert à des normes concurrentes, plus il est vulnérable. La sociologie constitue un champ très hétéronome, largement ouvert à des normes exogènes. Dès lors, les sociologues les moins acquis aux normes de l'activité scientifique sont tentés de s'assurer une reconnaissance plus rapide et plus visible sur «le second marché des intellectuels», pour s'exprimer comme Raymond Boudon (1990). Ils acquièrent de fait un intérêt personnel dans l'hétéronomie de leur propre domaine d'activité. Un cercle vicieux s'amorce alors. Ceux qui sont portés par les normes du monde médiatique acquièrent parmi leurs collègues une influence disproportionnée qui contribue encore à favoriser la domination des mœurs médiatiques. Dans un tel contexte, c'est la préoccupation de nouveauté - obsession des milieux journalistiques - qui l'emporte largement sur celle de cumulativité.

\section{EN CONCLUSION}

Si le défaut de cumulativité du savoir sociologique est indéniable, il appelle un jugement nuancé. Le progrès dans l'étude des conditions de la vie en société n'est certainement pas inexistant. Aux XVIII ${ }^{e}$ et $\mathrm{XIX}^{\mathrm{e}}$ siècles, pour les précurseurs de la sociologie institutionnalisée, la discipline de référence qui servait de modèle était la physique de l'époque. Les comportements et les états d'esprit de l'être humain semblaient pouvoir être analysés selon les principes de la mécanique. On cherchait alors des analogies entre les entités du système solaire unies par des forces d'attraction réciproque et les esprits humains. De nos jours, plus personne ne songerait à faire du mouvement des astres un modèle pour l'analyse des comportements humains. Au XIX ${ }^{e}$ siècle, comme le rappelait Goriely, la recherche d'un «facteur premier», d'un primat causal, était une préoccupation majeure (1950, p. 27). Dans la seconde moitié du XX'e siècle, Gurvitch se croyait encore tenu de polémiquer contre la théorie des «facteurs sociaux prédominants» (1963, Vol. 1, p. 1). A l'heure actuelle, la monocausalité ne fait plus recette, même si elle reste la tentation du sens commun. Les philanthropes du $\mathrm{XIX}^{\mathrm{e}}$ siècle étaient convaincus que l'isolement carcéral était bénéfique, qu'il amenait le condamné à méditer sur ses actes et à retrouver le droit chemin. On sait aujourd'hui que l'isolement carcéral est destructeur de la personnalité et que rien de positif ne peut en résulter. 
Il n'y a donc pas stagnation de la connaissance. On peut cependant se demander si les progrès enregistrés furent le résultat d'un processus de développement interne de la discipline ou si ce sont des facteurs exogènes qui se sont imposés. Le progrès de la connaissance sociologique ne correspond nullement à un processus linéaire fondé sur l'alternance de la recherche empirique et de l'élaboration théorique. Au plan sociétal, le «cercle piagétien» a pour conséquence qu'on observe une étroite correspondance entre l'évolution de la société et le développement de la sociologie. Il s'agit d'une «dialogique» au sens que Berthelot donne à ce terme, mais d'une dialogique globale. La sociologie est extrêmement sensible aux stimulations extérieures, aux idées en vogue, à l'air du temps. Elle y adapte son vocabulaire ainsi que ses procédés d'analyse, de démonstration et d'argumentation. Des conceptions théoriques et méthodologiques peuvent donner l'impression d'être caduques, non parce qu'elles ont épuisé leur potentiel d'analyse mais parce que les préoccupations de l'époque qui les a suscitées ont fait place à de nouvelles préoccupations, à de nouveaux problèmes.

A première vue, c'est dans le domaine de la comptabilité sociale au service de la prise de décision que le sentiment d'assister à un progrès est le plus perceptible. A l'heure actuelle, on dispose en effet dans tous les domaines d'une accumulation de données chiffrées qui permettent de faire le point de la situation et de se livrer à des extrapolations. Mais le progrès se limite au volume et à la qualité de l'information. Dès qu'il s'agit de son interprétation, le sentiment de progrès devient illusoire. En fait, c'est dans le domaine des applications à l'action qu'apparaît le plus nettement le fossé entre les acquis des sciences de la nature et les savoirs accumulés sur la vie en société. Le projet scientifique de la sociologie consiste à appliquer un maximum de systématique et de discipline intellectuelle à des faits de société qu'il est souvent très difficile de soustraire au règne des passions et des préjugés. Cette ambition à faire œuvre scientifique ne soutient néanmoins en aucune façon la comparaison avec les sciences de la nature auxquelles on se réfère généralement pour établir des critères de scientificité et d'où nous vient précisément l'aspiration à la cumulativité. Il serait bon que l'on cesse d'entretenir le mythe de la sociologie, science jeune qui pourra prétendre à un degré de scientificité comparable à celui des sciences de la nature lorsqu'elle aura atteint sa propre maturité. Il ne s'agit ni d'une question de degré ni d'une question de délai. Faute d'un réaménagement complet de son projet scientifique - difficilement imaginable en ce moment - la sociologie restera un genre hybride, mieux caractérisé par son rapport ambivalent aux classiques que par une parenté mythique avec les sciences de la nature.

Département de Sociologie, Université de Genève

\section{RÉFÉRENCES BIBLIOGRAPHIQUES}

Berthelot, Jean-Michel, Les vertus de l' incertitude, Paris, PUF, 1996.

Boudon, Raymond, L'analyse mathématique des faits sociaux, Paris, Plon, 1967.

Boudon, Raymond, L'idéologie. L'origine des idées reçues, Paris, Fayard, 1986.

Boudon, Raymond, «Les intellectuels et le second marché», in Revue européenne des sciences sociales, No 87, 1990, pp. 89-103 
Bourdieu, Pierre et al., La misère du monde, Paris, Seuil, 1993.

Bourdieu, Pierre, Raisons pratiques. Sur la théorie de l'action, Paris, Seuil/Points, 1994.

Bourdieu, Pierre, Sur la télévision, Paris, Liber Editions, 1996.

Busino, Giovanni, Critiques du savoir sociologique, Paris, PUF, Coll. « Sociologies», 1993.

Coenen-Huther, Jacques, Le fonctionnalisme en sociologie : et après? Bruxelles, Editions de l'Université de Bruxelles, 1984.

Coenen-Huther, Jacques, «Crise de la sociologie ou sociologues en crise?», in Revue européenne des sciences sociales, Tome XXXIII, No 100, 1995, pp. 185-189.

Coenen-Huther, Jacques, Tocqueville, Paris, PUF, Coll. «Que sais-je?», 1997.

Coenen-Huther, Jacques, «The Paths of Recognition: Boudon, Bourdieu, and the Second Market of Intellectuals », in: Graham C. Kinloch et Raj P. Mohan, Ideology and the Social Sciences, Westport, Conn., Greenwood, 2000, pp. 107-115.

Coenen-Huther, Jacques, «Le type idéal comme instrument de la recherche sociologique», in Revue française de sociologie, Vol. 44, No 3, 2003, pp. 531-547.

Coenen-Huther, Jacques, Sociologie des élites, Paris, Armand Colin, Coll. «Cursus», 2004a.

Coenen-Huther, Jacques, «La sociologie ou l'invention du social», in: Pascal Bridel, Ed., L'invention dans les sciences humaines. Hommage à Giovanni Busino, Genève, Labor et Fides, 2004b, pp. 241-252.

Corcuff, Philippe, Les nouvelles sociologies, Paris, Nathan, Coll. «128», 1995.

Dubois, Michel, Introduction à la sociologie des sciences, Paris, PUF, 1999.

Goldmann, Lucien, «Epistémologie de la sociologie», in: Piaget, Jean et al., Logique et connaissance scientifique, Paris, La Pléiade, 1967, pp. 992-1018.

Goriely, Georges, «De la relativité du savoir sociologique», in Revue de l'Institut de Sociologie, Bruxelles, No 1, 1950, pp. 19-34.

Gurvitch, Georges, La vocation actuelle de la sociologie, Tome I, édition revue, Paris, PUF, 1963.

Hirschhorn, Monique et Jacques Coenen-Huther, Eds, Durkheim et Weber. Vers la fin des malentendus? Paris, L'Harmattan, 1994.

Kuhn, Thomas, The Structure of Scientific Revolutions, 1962. Second edition, enlarged, Chicago, University of Chicago Press, 1970.

Lahire, Bernard, L' homme pluriel. Les ressorts de l'action, Paris, Nathan, 1998.

Lévi-Strauss, Claude, Anthropologie sociale deux, Paris, Plon, 1973.

Merton, Robert K., Social Theory and Social Structure, 1949. Enlarged edition, chap. I, On the History and Systematics of Sociological Theory, New York, The Free Press, 1968.

Mucchielli, Laurent, Mythes et histoire des sciences humaines, Paris, La Découverte, 2004.

Nisbet, Robert, The Sociological Tradition, Londres, Heinemann, 1966. Tr. fr. La tradition sociologique, Paris, PUF, Coll. «Quadrige», 1993.

Parsons, Talcott, The Structure of Social Action, 2 vol., New York, McGraw-Hill, 1937.

Parsons, Talcott, The Social System, 1951. Nouvelle édition, Londres, Routledge and Kegan Paul, 1970.

Parsons, Talcott, Introduction to the paperback edition of «The Structure of Social Action», New York, The Free Press, 1968, pp. V-XIV.

Parsons, Talcott, «Revisiting the Classics throughout a Long Career», in: Buford Rhea, Ed., The Future of the Sociological Classics, Londres, George Allen and Unwin, 1981, pp. 183-194.

Piaget, Jean, Etudes sociologiques, Genève, Droz, 1965.

Piaget, Jean, «Le système de la classification des sciences », in: Piaget, Jean et al., Logique et connaissance scientifique, Paris, La Pléiade, 1967, pp. 1151-1224.

Sorokin, Pitirim, Fads and Foibles in Modern Sociology, Chicago, Henry Regnery, 1956. Tr. fr.: Tendances et déboires de la sociologie américaine, Paris, Aubier, 1959.

Tenbruck, Friedrich H., «Die Genesis der Methodologie Max Webers», in Kölner Zeitschrift für Soziologie und Sozialpsychologie, Vol. 11, 1959, pp. 573-630. 